\title{
Portfolio Selection under Condition of Variable Weights
}

\author{
Reza Keykhaei, Mohammad Taghi Jahandideh \\ College of Mathematical Sciences, Isfahan University of Technology, Isfahan, Iran \\ Email: r.keykhaei@math.iut.ac.ir, jahandid@cc.iut.ac.ir
}

Received July 11, 2012; revised August 9, 2012; accepted August 16, 2012

\begin{abstract}
In this paper we generalize the single-period Markowitz Mean-Variance portfolio selection problem. The Markowitz's model requires that after choosing the number of each security which constructs the portfolio in the beginning of the investment period, these numbers remain constant during and at the end of the investment period. We drop this assumption and consider an investment model in which the number of each security can vary randomly during the investment period. Indeed we consider a single-period investment with the property that the initial weight of each security is not equal to the final weight of that security. We redefine the notion of the rate of return of each security and show that the return of the investment in a cash account is not certain. We investigate some alternatives among risky securities which acts similar to cash accounts. For this we introduce the notion of free security and relate free securities to a riskless security.
\end{abstract}

Keywords: Mean-Variance Optimization; Riskless Asset; Efficient Frontier; Free Asset

\section{Introduction}

After that Markowitz [1] originated the Modern Portfolio Theory (MPT) by introducing his single-period MeanVariance (M-V) portfolio selection problem in 1952, many authors have worked on this field. Markowitz considered a portfolio of risky asset. In 1958 Tobin [2] introduced the notion of riskless asset when he entered cash account in his portfolio and stated the one-fund theorem. This theorem says that when both borrowing and lending at the riskless rate are allowed, there is a single risky fund such that every efficient portfolio (portfolios whose expected returns are largest among all portfolios with the same variance) can be constructed as a combination of the fund and the riskless asset. In the Mean-Standard Deviation space, the efficient portfolios can be represented by a straight line which is called the optimal Capital Allocation Line (CAL) (see Tütüncü [3]). In 1972, Merton [4] described the mathematics of the efficient portfolios in full analytic approach. The geometric viewpoint of the Mean- Variance analysis was developed by Merton [4] and Roll [5]. There are a huge contributions about asset allocation problem. Recently Steinbach [6] in a survey paper has a complete review on the different models in this case.

In the classic one-period $\mathrm{M}-\mathrm{V}$ portfolio selection problem, the weight assigned to each security contained in portfolio has been considered to be constant. In other words, if someone at the beginning of the investment period $(t=0)$ enters in the capital market with initial wealth $X(0)$ and invest his/her wealth as

$$
X(0)=\sum_{i=1}^{n} \phi_{i} S_{i}(0)
$$

between $n$ securities, then his/her wealth at the end of the investment period $(t=T)$ is

$$
X(T)=\sum_{i=1}^{n} \phi_{i} S_{i}(T),
$$

where $S_{i}(0)$ and $S_{i}(T)$ are, respectively, the value of the $i$ th security at the beginning and the end of the period, and for each $i, \phi_{i}$ is the number of shares of the $i$ th security. Here, $S_{i}(T)$ is nondeterministic and is considered to be a random variable, although, at the beginning and at the end of the investment period, $\phi_{i}{ }^{\prime} \mathrm{s}$ remain fixed.

In our model, we assume that $\phi_{i}$ 's are nonconstant and vary randomly. In fact it is possible that $\phi_{i}(0) \neq \phi_{i}(T)$ for some $i$, where for each $i, \phi_{i}(0)$ is the number of shares of the $i$ th security at the beginning of the period and $\phi_{i}(T)$ is the number of the same one at the end of the period. Thus the value of portfolio at the beginning of the period is

$$
X(0)=\sum_{i=1}^{n} \phi_{i}(0) S_{i}(0)
$$

and at the end of the period, it is

$$
X(T)=\sum_{i=1}^{n} \phi_{i}(T) S_{i}(T) .
$$

We can interpret the changes in the values of the $\phi_{i}$ 's as follows. Consider an investor who has this opportunity to receive some amount of the $i$ th security, as a gift or a 
reward, during the investment period (after constituting his initial portfolio), it is possible that he/she loses some amount of the $i$ th security by a contract that force $\mathrm{him} /$ her to pay his obligation via the $i$ th security. This variability is more plausible in the commodity markets. One who intends to invest his/her wealth on the commodities, some activities such as holding and transporting of commodities impose additional risks; besides other commodity risks, caused by natural or accidental events, on the investment. If we consider the occurrence of the above events as a possibility, then the value of $\phi_{i}(T)$ will be a random variable.

The importance of our model can be seen in the following conditions:

- There is someone who is willing or is forcing to make an investment by imposing some obligations in his/her portfolio investment;

- There might be no possibility or willing to insure the commodities included in the investment;

- Moreover, since this model generalizes the Markowitz model, we can use the obtained results in the classic portfolio selection problems.

It is important to note that although the essence of $S_{i}(T)$, as a random variable, is common between all investors, but the nature of $\phi_{i}(T)$ is special for each investor and can vary from one investor to another. Thus we fit our model to a special investor who wants to construct the optimal portfolio by considering the fact that the security weights can vary randomly.

Under this new assumption, we want to develop the notion of one-period Markowitz portfolio selection problem. On the other hand, we know that in the classic MeanVariance analysis (with invariable weights), the rate of return of each security $r_{i}$ and the rate of return of the portfolio $r$ are defined. Then the mean and the variance of $r$ are set the criteria for choosing the optimal portfolio. Indeed $r_{i}$ and $r$ are defined as follow:

$$
r_{i}=\frac{S_{i}(T)-S_{i}(0)}{S_{i}(0)}, \quad i=1, \cdots, n
$$

and

$$
r=\frac{X(T)-X(0)}{X(0)},
$$

then

$$
\begin{aligned}
r & =\frac{\sum_{i=1}^{n} \phi_{i} S_{i}(T)-\sum_{i=1}^{n} \phi_{i} S_{i}(0)}{X(0)} \\
& =\sum_{i=1}^{n} \frac{\phi_{i} S_{i}(0)}{X(0)} \frac{S_{i}(T)-S_{i}(0)}{S_{i}(0)} \\
& =\sum_{i=1}^{n} x_{i} r_{i},
\end{aligned}
$$

in which

$$
x_{i}=\frac{\phi_{i} S_{i}(0)}{X(0)}, \quad i=1, \cdots, n
$$

Clearly

$$
\sum_{i=1}^{n} x_{i}=1 .
$$

Indeed for each $i, x_{i}$ is the the assigned weight allocated to the $i$ th security in the portfolio. Here we denote each security by its weight and the portfolio by the vector of security weights. Thus $x_{i}$ and

$$
\mathbf{X}:=\left(x_{1}, \cdots, x_{n}\right)^{\prime} \in R^{n}
$$

denote the $i$ th security and the portfolio, respectively. Obviously in the case that $\phi_{i}$ 's are invariable, $x_{i}$ 's are also invariable and for variable $\phi_{i}$ 's, $x_{i}$ 's are variable. The M-V portfolio selection problem minimizes the variance of $r$, subject to a desired mean return $\rho$. Indeed the problem leads to the following quadratic programming problem which its solution is known:

Problem $(\star)$.

$$
\begin{gathered}
\min _{\mathbf{X}} \frac{1}{2} \mathbf{X}^{\prime} \Sigma \mathbf{X} \\
\text { s.t. } \quad \mathbf{1}^{\prime} \mathbf{X}=1, \\
\overline{\mathbf{R}}^{\prime} \mathbf{X}=\rho,
\end{gathered}
$$

where $\Sigma$ is the covariance matrix of the security returns and $\overline{\mathbf{R}}$ is the vector of mean returns $\left(\bar{r}_{1}, \cdots, \bar{r}_{n}\right)^{\prime}$. Also $\mathbf{1}$ is the $n$-column vector of ones.

This paper is organized as follows: In Section 2, we introduce the model and its formulation. The connection between free securities and riskless assets is investigated in Section 3. In Section 4, we describe the relation between a set of free securities with both a free security and a riskless security.

\section{Portfolio with Variable Weights}

Now we want to know how we should state the portfolio selection problem with variable weights so that we can apply the standard methods to solve it (see Theorem 2.5). To do this, we show that, if an investment asset has any additional benefits beyond capital gains such as random growths or losses of shares, then returns should be adjusted to include any additional components of returns. As the first step, it is necessary to determine the increments of the number of shares for each security.

Definition 2.1. For a portfolio with variable weights, let for each $i$,

$$
\lambda_{i}=\frac{\phi_{i}(T)-\phi_{i}(0)}{\phi_{i}(0)},
$$

where $\phi_{i}(0)$ and $\phi_{i}(T)$ are the number of security $x_{i}$ 
at the beginning and at the end of the period, respectively. We call $\lambda_{i}$ the rate of increments of $x_{i}$.

Example 2.2. Assume that an event occurs according to a Poisson process $\{N(t): t \geq 0\}$ with rate $\lambda$, and for any arrival, the investor obligate to pay one share of the $i$ th security, where $i$ is fixed. Then at the end of the investment period $(t=T)$, with $N(T)=k$,

$$
\phi_{i}(T)=\phi_{i}(0)-k, \quad k=0,1,2, \cdots .
$$

Thus $\lambda_{i}$ is a nonpositive random variable such that

$$
\begin{aligned}
P\left(\lambda_{i}=l\right) & =P\left(\phi_{i}(T)=\phi_{i}(0)-k\right) \\
& =P(N(T)=k) \\
& =e^{-\lambda T} \frac{(\lambda T)^{k}}{k !},
\end{aligned}
$$

for $l=-k / \phi_{i}(0)$, where $k=0,1,2, \cdots$.

By applying the above definition and (2), we have

$$
\begin{aligned}
r= & \frac{\sum_{i=1}^{n} \phi_{i}(T) S_{i}(T)-\sum_{i=1}^{n} \phi_{i}(0) S_{i}(0)}{X(0)} \\
= & \sum_{i=1}^{n} \frac{\phi_{i}(0) S_{i}(0)}{X(0)} \frac{S_{i}(T)-S_{i}(0)}{S_{i}(0)} \\
& +\sum_{i=1}^{n} \frac{\phi_{i}(0) S_{i}(0)}{X(0)} \frac{\phi_{i}(T)-\phi_{i}(0)}{\phi_{i}(0)} \frac{S_{i}(T)}{S_{i}(0)} \\
= & \sum_{i=1}^{n} x_{i} r_{i}+\sum_{i=1}^{n} x_{i} \lambda_{i}\left(1+r_{i}\right) \\
= & \sum_{i=1}^{n} x_{i}\left(r_{i}+\lambda_{i}\left(1+r_{i}\right)\right),
\end{aligned}
$$

for

$$
x_{i}=\left(\phi_{i}(0) S_{i}(0)\right) / X(0)
$$

as the initial weight allocated to the $i$ th security. $r$ can be written as

$$
r=\sum_{i=1}^{n} x_{i} R_{i}
$$

where

$$
R_{i}:=r_{i}+\lambda_{i}\left(1+r_{i}\right) .
$$

Definition 2.3. Let for each $i=1, \cdots, n, r_{i}$ be the rate of the return of the security $x_{i}$. We define $u_{i}:=1+R_{i}$ and

$$
R:=\sum_{i=1}^{n} x_{i} u_{i}
$$

as the total-return of $x_{i}$ and the total-return (or briefly the return) of the portfolio, respectively.

Note. Suppose we possess one share of the $i$ th security. By considering its total-return $u_{i}$, at the end of the period, its value becomes

$$
S_{i}(0) u_{i}=S_{i}(0)\left(1+\lambda_{i}\right)\left(1+r_{i}\right),
$$

as expected, since $1+\lambda_{i}$ is the number of this security. Also, $X(T)=R X(0)$.

Now we can state our portfolio selection problem in the form of the problem $(\star)$. Assume that all the securities are risky and $\mathbf{U}$ is the $n$-column random vector and $\Sigma^{*}$ is the covariance matrix of the totalreturns $u_{1}, \cdots, u_{n}$. Also, define $\overline{\mathbf{U}}:=\mathbb{E}(\mathbf{U})$ as the $n$-column vector of mean total-returns $\bar{u}_{1}, \cdots, \bar{u}_{n}$ of these $n$ securities. The mean and covariance matrix of the totalreturns are assumed to be known. By these assumptions, the portfolio selection problem with variable weights can be stated in the following form:

Problem 1. Consider the desired mean total-return $\rho$ for the portfolio. Our aim is to solve the following Mean-Variance portfolio selection problem,

$$
\begin{gathered}
\min _{\mathbf{X}} \frac{1}{2} \mathbf{X}^{\prime} \Sigma^{*} \mathbf{X} \\
\text { s.t. } \quad \mathbf{1}^{\prime} \mathbf{X}=1, \\
\overline{\mathbf{U}}^{\prime} \mathbf{X}=\rho .
\end{gathered}
$$

Assumptions. The imposed assumptions on the problem are as follows:

- (A.1) The covariance matrix $\Sigma^{*}$ is positive definite.

- (A.2) The mean vector $\overline{\mathbf{U}}$ is not a multiple of $\mathbf{1}$.

The following constants are frequently used in the sequel and we define them similar to those defined in [6] as follows:

$$
\begin{aligned}
& \alpha:=\mathbf{1}^{\prime} \Sigma^{*-1} \mathbf{1}, \quad \beta:=\mathbf{1}^{\prime} \Sigma^{*-1} \overline{\mathbf{U}}, \\
& \gamma:=\overline{\mathbf{U}}^{\prime} \Sigma^{*-1} \overline{\mathbf{U}}, \quad \delta:=\alpha \gamma-\beta^{2} .
\end{aligned}
$$

Lemma 2.4. The constants $\alpha, \gamma$ and $\delta$ are positive.

Proof. See Lemma 1.3 of [6].

In the above lemma we realized that we can not specify the exact rang of $\beta$. Indeed $\beta$ may not be positive.

By applying the Lagrangian multiplier method, we can interpret the solution of Problem 1. In the following we display the primal-dual solution of Problem 1 by $\left(\mathbf{X}^{*}, \lambda^{*}, \mu^{*}\right)$. Also $\sigma^{2}(\rho)$ denotes the optimal variance of the problem, which is the variance of the solution. Like [6], we call the whole graph of the optimal variance the efficient frontier.

Theorem 2.5. Problem 1 has the unique primal-dual solution

$$
\begin{aligned}
& \mathbf{X}^{*}=\Sigma^{*-1}\left(\lambda^{*} \mathbf{1}+\mu^{*} \overline{\mathbf{U}}\right), \\
& \lambda^{*}=(\gamma-\beta \rho) / \delta, \\
& \mu^{*}=(\alpha \rho-\beta) / \delta .
\end{aligned}
$$

Also the optimal variance is

$$
\sigma^{2}(\rho)=\lambda^{*}+\mu^{*} \rho=\left(\alpha \rho^{2}-2 \beta \rho+\gamma\right) / \delta,
$$


Proof. See Theorem 1.5 and Theorem 1.7 of [6].

Note. Although it seems that the variability of the weights complicates the portfolio selection problem, we see that the problem can be converted to the classical form of portfolio selection problem with invariable weights (Problem $(\star)$ ) by using the notion of totalreturns. Therefore, we can employ most of the results for portfolio selection in the situation of invariable weights to the case of variable weights. Clearly when

$$
\lambda_{i}^{i i d} \equiv 0^{1}
$$

for each $i=1, \cdots, n$, then we have a portfolio selection problem with invariable weights. Thus our model can be interpreted as an extension of classical $\mathrm{M}-\mathrm{V}$ optimization problem.

\section{Existence of Riskless Securities}

Note that a security is riskless, if its return is guaranteed and hence it is deterministic. Although cash accounts are commonly considered as riskless securities, in our model they are not riskless. Because of the change in weights, the amount of the gain for a cash account is not deterministic. Indeed the variance of its total-return is not zero. Now we redefine the notion of riskless security on the bases of its total-return.

Definition 3.1. We say a security is riskless, if the variance of its total-return is zero and denote it by its weight $x_{f_{0}}$.

By the above definition, a cash account is riskless if and only if the rate of its increments is constant. In addition, it is not necessary for a riskless security to be of the form of a cash account.

As mentioned above, in our model, it is rare that a portfolio contains a riskless security. In the following, we try to find some risky securities which act like riskless securities to improve our model. Clearly this can also be applied for $\mathrm{M}-\mathrm{V}$ portfolio selection with invariable weights.
Definition 3.2. Let the total-return of a risky security be uncorrelated with the total-return of all securities in a set of risky securities $S$. We call such a security free (with respect to $S$ ) or S-free; and display it and its total-return by $x_{f}$ and $u_{f}$, respectively.

Example 3.3. We investigate the correlation coefficients between the weekly rate of returns of a set of stocks chosen from S \& P500: AEP, ARG, BMY, CPB, DPS, DUK, ERTS, LH, LLY, MCD, MKC, PGN, PPL, SCG, SO, SWY, UNH, WAG, WAT, WDC, WEC, WFV, WLP, WPI, WPO, WYNN, YHOO, YUM, ZION, ZMH. The data consist of daily closing stock prices over 2 years period from 3/12/2009 to 3/11/2011. We choose two assets ARG and WEC, which have low correlation coefficients with the other assets and present the correlation coefficients between their rate of returns and the rate of returns of the other assets in Table 1.

Suppose we can construct a portfolio for which the rate of increments of each asset has a mean in $[-1,0]$, is independent of the rate of return of that asset and it is independent of the rate of increments and the rate of returns of the other assets. Let us say two random variables are uncorrelated if the absolute value of their estimated correlation coefficient is less than 0.1 (at a significant level grater than 0.05). Now we can observ from Table 1 that for a weekly investment, the stock WEC is $S$-free, where $S=$ AARG, BMY, CPB, DPS, LH, MKC, PPL, UNH, WAT, WDC, WFV, WLP, WPI, WYNN, YHOO, YUM, ZION, ZMH $\}$, since, for WEC with the total-return $u_{w}$ and the $i$ th asset of $S$ with the total return $u_{i}$, we have

$$
\begin{aligned}
& \operatorname{Cov}\left(u_{w}, u_{i}\right) \\
= & \operatorname{Cov}\left(\left(1+\lambda_{w}\right)\left(1+r_{w}\right),\left(1+\lambda_{i}\right)\left(1+r_{i}\right)\right) \\
= & \left(1+\bar{\lambda}_{w}\right)\left(1+\bar{\lambda}_{i}\right) \operatorname{Cov}\left(r_{w}, r_{i}\right) \\
& +\left(1+\bar{r}_{w}\right)\left(1+\bar{r}_{i}\right) \operatorname{Cov}\left(\lambda_{w}, \lambda_{i}\right) \\
= & \left.\left(1+\bar{\lambda}_{w}\right)\left(1+\bar{\lambda}_{i}\right)\right) \operatorname{Cov}\left(r_{w}, r_{i}\right),
\end{aligned}
$$

\begin{tabular}{|c|c|c|c|c|c|c|c|c|c|c|}
\hline & AEP & $\mathrm{ARG}$ & BMY & $\mathrm{CPB}$ & DPS & DUK & ERTS & LH & LLY & MCD \\
\hline WEC & $0.231^{* *}$ & 0.059 & 0.038 & 0.084 & 0.038 & $0.216^{* *}$ & $0.089^{*}$ & 0.044 & $0.090^{*}$ & $0.141^{* *}$ \\
\hline \multirow[t]{2}{*}{ ARG } & 0.06 & 1 & $0.093^{*}$ & $0.097^{*}$ & $0.128^{* *}$ & $0.099^{*}$ & $0.184^{* *}$ & 0.042 & 0.086 & 0.073 \\
\hline & $\mathrm{MKC}$ & PGN & PPL & SCG & SO & SWY & UNH & WAG & WAT & WEC \\
\hline WEC & -0.052 & $0.211^{* *}$ & 0.055 & $0.288^{* *}$ & $0.232^{* *}$ & $0.104^{*}$ & 0.016 & $0.107^{*}$ & 0.065 & 1 \\
\hline \multirow[t]{2}{*}{$\mathrm{ARG}$} & -0.019 & 0.024 & $0.183^{* *}$ & $0.091^{*}$ & 0.074 & 0.08 & $0.109^{*}$ & 0.071 & $0.283^{* *}$ & 0.059 \\
\hline & WDC & WFV & WLP & WPI & WPO & WYNN & YHOO & YUM & ZION & $\mathrm{ZMH}$ \\
\hline WEC & 0.042 & 0.049 & 0.028 & 0.051 & $0.100^{*}$ & 0.055 & 0.046 & 0.084 & 0.073 & 0.073 \\
\hline $\mathrm{ARG}$ & $0.281^{* *}$ & $0.165^{* *}$ & 0.025 & 0.075 & $0.094^{*}$ & $0.196^{* *}$ & 0.196 & $0.134^{* *}$ & $0.132^{* *}$ & $0.182^{* *}$ \\
\hline
\end{tabular}

Table 1. Estimated values of correlation coefficients.

${ }^{1}$ Iid means independent and identically distributed. 
where $\quad \bar{r}_{w}=\mathbb{E}\left(r_{w}\right), \quad \bar{\lambda}_{w}=\mathbb{E}\left(\lambda_{w}\right), \quad \bar{r}_{i}=\mathbb{E}\left(r_{i}\right) \quad$ and $\bar{\lambda}_{i}=\mathbb{E}\left(\lambda_{i}\right)$. So,

$$
\left|\operatorname{Cov}\left(u_{w}, u_{i}\right)\right| \leq\left|\operatorname{Cov}\left(r_{w}, r_{i}\right)\right| .
$$

Also, the stock ARG is $S$-free, where $S=\{$ AEP, LH, MCD, MKC, PGN, SWY, SO, WAG, WEC, WLP, WPI $\}$. The correlation coefficients denoted by ${ }^{*}$ and ${ }^{* *}$ are significant at a (2-tailed) level less than or equal to 0.05 and 0.01 , respectively. In the other cases the significant levels are grater than 0.05 .

\subsection{Portfolio with Risky Securities and One Free Security}

Let a portfolio consists of $n \geq 1$ risky securities $x_{1}, \cdots, x_{n}$ and a free security $x_{f}$. Indeed the portfolio can be presented as $\mathbf{X}_{1}:=\left(\mathbf{X}^{\prime}, x_{f}\right)^{\prime} \in R^{n+1}$, where $\mathbf{X}=\left(x_{1}, \ldots, x_{n}\right)^{\prime}$. For convenience, throughout the paper, we sperate the free securities from other risky securities and call $\mathbf{X}$ and $x_{f}$ the "risky part" and the "free part" of the portfolio and denote the portion of each part that appears in the primal solution by "risky solution" and "free solution", respectively. Let $\mathbf{U}_{1}=\left(u_{1}, \cdots, u_{n}, u_{f}\right)$ ' be the random vector of total-returns and $\Sigma_{1}^{*}$ be their covariance matrix. Also let

$$
\overline{\mathbf{U}}_{1}:=\mathbb{E}\left(\mathbf{U}_{1}\right)=\left(\bar{u}_{1}, \ldots, \bar{u}_{n}, \bar{u}_{f}\right)^{\prime}
$$

and $\mathbf{1}_{1}$ be the $(n+1)$-column vectors of ones. In the sequel $\Sigma^{*}$ and $\overline{\mathbf{U}}$ refer to the risky part of the portfolio.

Now our situation is a special case of Problem 1 with one additional risky security $x_{f}$ and it can be stated as a new problem:

Problem 2.

$$
\begin{gathered}
\min _{\mathbf{X}, x_{f}} \frac{1}{2} \mathbf{X}_{1^{\prime}} \Sigma_{1}^{*} \mathbf{X}_{1} \\
\text { s.t. } \quad \mathbf{1}_{1^{\prime}} \mathbf{X}_{1}=1, \\
\overline{\mathbf{U}}_{1^{\prime}} \mathbf{X}_{1}=\rho .
\end{gathered}
$$

Assumptions. Here the assumptions are (A.1) and

- (A.3) The mean vector $\overline{\mathbf{U}}_{1}$ is not a multiple of $\mathbf{1}_{1}$. Note. We fix these assumption throughout the paper. Let

$$
\sigma_{f}^{2}:=\operatorname{Var}\left(u_{f}\right)
$$

and

$$
s:=\left(\sigma_{f}^{2}\right)^{-1}
$$

We define the corresponding constants which are

${ }^{2}$ This notation is similar to $\delta^{c}$ in [6], when $u$ is considered to be a return of a cash account.

${ }^{3}$ Explicitly we suppose that $u$ is a constant. stated in (4) by:

$$
\begin{aligned}
& \alpha_{s}:=\mathbf{1}_{1}^{\prime} \Sigma_{1}^{*-1} \mathbf{1}_{1}, \quad \beta_{s}:=\mathbf{1}_{1}^{\prime} \Sigma_{1}^{*-1} \overline{\mathbf{U}}_{1}, \\
& \gamma_{s}:=\overline{\mathbf{U}}_{1}^{\prime} \Sigma_{1}^{*-1} \overline{\mathbf{U}}_{1}, \quad \delta_{s}:=\alpha_{s} \gamma_{s}-\beta_{s}^{2} .
\end{aligned}
$$

Obviously

$$
\Sigma_{1}^{*-1}=\left(\begin{array}{cc}
\Sigma^{*-1} & 0 \\
0 & s
\end{array}\right) .
$$

Now

$$
\begin{aligned}
& \alpha_{s}=\alpha+s, \quad \beta_{s}=\beta+s u, \\
& \gamma_{s}=\gamma+s u^{2}, \quad \delta_{s}=\delta+s \delta_{f}
\end{aligned}
$$

where $u:=\bar{u}_{f}$ and

$$
\delta_{f}:=\alpha u^{2}-2 \beta u+\gamma^{2},
$$

and $\alpha, \beta, \gamma$ and $\delta$ are related to the risky part of the portfolio and are denoted by (4).

First, consider $\sigma_{f}^{2}$ as a variable, what will happen when $\sigma_{f}^{2}$ decreases to zero or equivalently when $s$ increases to infinity. More precisely, what is the behavior of the solution or the efficient frontier of Problem 2 when the variance of $x_{f}$ tends to zero. Although in our model there is no riskless cash account, by choosing assets with small variances, we show that one can get similar results in contrast with the case that we have riskless cash account. That is, the solution and the optimal variance of the problem converge to the solution and optimal variance in the case where the portfolio contains riskless security. Then with a little connivance, instead of a riskless security, we can use a risky (free) security such that its total-return has the same mean with the cash account and its variance is close to zero. In fact if we consider $\sigma_{f}^{2}$ as a variable, then the primal-dual solution and the efficient frontier are functions of the variable $\sigma_{f}^{2}$ and to reach our aim, it is sufficient to show that they are continuous functions of $\sigma_{f}^{2} \in[0, \infty)$. First, we investigate the solution of problem 2 when $\sigma_{f}^{2}=0$.

Theorem 3.4. Let $\sigma_{f}^{2}=0$. Then, Problem 2 has the primal-dual solution as follows:

$$
\begin{gathered}
\lambda=-u \mu, \quad \mu=(\rho-u) / \delta_{f} . \\
\mathbf{X}=\Sigma^{*-1}(\lambda \mathbf{1}+\mu \overline{\mathbf{U}}), \quad x_{f_{0}}=1-\mu(\beta-u \alpha) .
\end{gathered}
$$

Also, the optimal variance is

$$
(\rho-u)^{2} / \delta_{f}=\lambda+\mu \rho .
$$

Proof. See Theorem 1.8 of [6].

Theorem 3.5. The primal-dual solution and optimal variance of Problem 2 are continuous with respect to $\sigma_{f}^{2} \in[0, \infty)^{3}$.

Proof. Let $\sigma_{f}^{2} \neq 0$. By Theorem 2.5, the corresponding primal-dual solution of the problem for each $s=\left(\sigma_{f}^{2}\right)^{-1}$ is 


$$
\begin{gathered}
\left(\mathbf{X}_{s^{\prime}}, x_{f_{s}}\right)^{\prime}=\Sigma_{1}^{*-1}\left(\lambda_{s} \mathbf{1}_{1}+\mu_{s} \overline{\mathbf{U}}_{1}\right), \\
\lambda_{s}=\left(\gamma_{s}-\beta_{s} \rho\right) / \delta_{s}, \mu_{s}=\left(\alpha_{s} \rho-\beta_{s}\right) / \delta_{s}
\end{gathered}
$$

Then,

$$
\mathbf{X}_{s}=\Sigma^{*-1}\left(\lambda_{s} \mathbf{1}+\mu_{s} \overline{\mathbf{U}}\right), x_{f_{s}}=s\left(\lambda_{s}+u \mu_{s}\right) .
$$

Also the optimal variance is

$$
\sigma_{s}^{2}(\rho)=\lambda_{s}+\mu_{s} \rho .
$$

By Lemma 2.4, $\delta_{s}$ is positive. Thus the assertion holds for $\sigma_{f}^{2} \in(0, \infty)$.

Let $\sigma_{f}^{2}=0$. By Theorem 3.4, Problem 2 has the primal-dual solution

$$
\begin{gathered}
\lambda=-u \mu, \quad \mu=(\rho-u) / \delta_{f} . \\
\mathbf{X}=\Sigma^{*-1}(\lambda \mathbf{1}+\mu \overline{\mathbf{U}}), x_{f_{0}}=1-\mu(\beta-u \alpha),
\end{gathered}
$$

and the optimal variance

$$
(\rho-u)^{2} / \delta_{f}=\lambda+\mu \rho .
$$

To verify the assertion at $\sigma_{f}^{2}=0$, substitute the values in (7) in Equations (9)-(11) and take limit when $\sigma_{f}^{2}$ goes to zero or equivalently when $S$ goes to infinity and compar the results with Equations (12)-(14). Now the assertion holds.

Corollary 3.6. Let a portfolio of risky securities contains a cash account $x_{c}$ for which its rate of increments $\lambda_{c}$ is uncorrelated with the total-return of the other securities. If the variance of $\lambda_{c}$ converges to zero, then the primal-dual and the optimal variance of the $M-V$ portfolio selection problem converges to the same values for which $x_{c}$ is replaced by a riskless cash account $x_{c}$ with the same mean total-return.

Proof. Assume $\left(x_{1}, \cdots, x_{n}, x_{c}\right)$ is the portfolio. Also, let $r_{c}$, and $u_{c}$ be the rate of return and total-return of $x_{c}$, respectively. Then

$$
\begin{aligned}
\operatorname{cov}\left(u_{i}, u_{c}\right) & =\operatorname{cov}\left(u_{i}, 1+\lambda_{c}\right)\left(1+r_{c}\right) \\
& =\left(1+r_{c}\right) \operatorname{cov}\left(u_{i}, 1+\lambda_{c}\right)=0,
\end{aligned}
$$

for $i=1, \cdots, n$. Thus $x_{c}$ is a free asset and the assertion holds by Theorem 3.5.

From now on, suppose $\left(\mathbf{X}_{0}^{* 1}, x_{f_{0}}^{*}\right)$ and $\sigma_{0}^{2}(\rho)$ are the primal solution and optimal variance of Problem 2 when $\sigma_{f}^{2}=0$, respectively (see Equations (13) and (14)).

\subsection{Portfolio with Risky Securities: A Free Security and a Guaranteed Total Loss}

Following section 1.3 of [6], consider a portfolio with $n \geq 1$ risky securities $x_{1}, \cdots, x_{n}$ and a security $x_{l}$ with guaranteed total loss, i.e. $u_{l} \equiv \mathbb{E}\left(u_{l}\right)=0$. In this case the portfolio will be $\hat{\mathbf{X}}:=\left(\mathbf{X}^{\prime}, x_{l}\right)^{\prime} \in R^{n+1}$, where $\mathbf{X}$ is the risky part of the portfolio. Since $x_{l}$ is not risky then all covariances associated with $x_{l}$ vanishes. Then the mean and the variance of the total-return of the portfolio become $\overline{\mathbf{U}}^{\prime} \mathbf{X}$ and $\mathbf{X}^{\prime} \boldsymbol{\Sigma}^{*} \mathbf{X}$, respectively. To eliminate arbitrage opportunity, we impose the additional constraint $x_{l} \geq 0$. With this conditions the problem can be stated as follows:

Problem 3.

$$
\begin{gathered}
\min _{\mathbf{X}, x_{l}} \frac{1}{2} \mathbf{X}^{\prime} \Sigma^{*} \mathbf{X} \\
\text { s.t. } \quad \mathbf{1}_{1^{\prime}} \hat{\mathbf{X}}=\mathbf{1}^{\prime} \mathbf{X}+x_{l}=1, x_{l} \geq 0 \\
\overline{\mathbf{U}}^{\prime} \mathbf{X}=\rho .
\end{gathered}
$$

We apply the Karush-Kuhn-Tucker conditions to solve the above problem. To do this, let $\eta$ be the multiplier of the nonnegativity constraint $x_{1} \geq 0$.

Theorem 3.7. Problem 3 has the following unique primal-dual solution:

1) If $\beta>0$, then

a) For $\rho>\gamma / \beta, x_{l}=0, \eta=-\lambda>0$, and $\mathbf{X}, \lambda, \mu$ are the same as in the primal-dual solution of Problem 1.

b) For $\rho \leq \gamma / \beta$,

$$
\mathbf{X}=\mu \Sigma^{*-1} \overline{\mathbf{U}}, x_{l}=1-\mu \beta, \mu=\frac{\rho}{\gamma}, \eta=\lambda=0 .
$$

2) If $\beta \leq 0$, then

$$
\mathbf{X}=\mu \Sigma^{*-1} \overline{\mathbf{U}}, x_{l}=1-\mu \beta, \mu=\frac{\rho}{\gamma}, \eta=\lambda=0 .
$$

Note that the optimal variance in conditions 1) b) and 2) is

$$
\mathbf{X}^{\prime} \Sigma^{*} \mathbf{X}=\mu^{2} \overline{\mathbf{U}}^{\prime} \Sigma^{*-1} \overline{\mathbf{U}}=\frac{\rho^{2}}{\gamma}
$$

Proof. The Lagrangian associated with Problem 3 is

$$
\begin{aligned}
& L\left(\mathbf{X}, x_{l}, \lambda, \eta\right) \\
= & \frac{1}{2} \mathbf{X}^{\prime} \Sigma^{*} \mathbf{X}-\lambda\left(\mathbf{1}^{\prime} \mathbf{X}+x_{l}-1\right) \\
& -\mu\left(\overline{\mathbf{U}}^{\prime} \mathbf{X}-\rho\right)-\eta x_{l} .
\end{aligned}
$$

We can consider the system of necessary conditions for the problem as follow:

$$
\begin{aligned}
& \left(\begin{array}{cccc}
\Sigma^{*} & 0 & \mathbf{1} & \overline{\mathbf{U}} \\
0 & 0 & 1 & 0 \\
\mathbf{1}^{\prime} & 1 & 0 & 0 \\
\overline{\mathbf{U}}^{\prime} & 0 & 0 & 0
\end{array}\right)\left(\begin{array}{c}
\mathbf{X} \\
x_{l} \\
-\lambda \\
-\mu
\end{array}\right)=\left(\begin{array}{l}
0 \\
\eta \\
1 \\
\rho
\end{array}\right), \\
& x_{l} \geq 0, \quad \eta \geq 0, \quad \eta x_{l}=0 .
\end{aligned}
$$

Solving the above system results in $\lambda=-\eta$ and

$$
\mathbf{X}=\Sigma^{*-1}(\mu \overline{\mathbf{U}}-\eta \mathbf{1})
$$

Consider the condition $\eta x_{l}=0$. If $\eta=0$, then we have 
$\lambda=0$ and

$$
\mathbf{X}=\mu \Sigma^{*-1} \overline{\mathbf{U}}, \quad x_{l}=1-\mu \mathbf{1}^{\prime} \Sigma^{*-1} \overline{\mathbf{U}}=1-\mu \beta .
$$

Thus

$$
\rho=\overline{\mathbf{U}} \mathbf{X}=\mu \overline{\mathbf{U}} \Sigma^{*-1} \overline{\mathbf{U}}=\mu \gamma
$$

So

$$
x_{l}=1-\rho \frac{\beta}{\gamma} .
$$

If $x_{l}=0$, then $\lambda=(\gamma-\rho \beta) / \delta$ as in Problem 1 . Then for $\beta \neq 0$,

$$
\rho=\frac{\gamma}{\beta}-\lambda \frac{\delta}{\beta}
$$

Note that $\gamma$ and $\delta$ are positive by Lemma 2.4. Now the proof of the Theorem:

Let $\beta>0$, and let $\rho>\gamma / \beta$. If $\eta=0$, then $x_{l}$ is negative by (16). On the other hand, if $x_{l}=0$, then by (17) the value of $\lambda$ will be negative, and hence $\eta$ is positive. Also for $\rho<\gamma / \beta, x_{l}=0$ yields that $\lambda$ is positive and therefore $\eta$ is negative, whereas when $\eta=0, x_{l}$ is positive. For $\rho=\gamma / \beta, \eta=x_{l}=0$. So part (1) holds.

Let $\beta<0$, since we assume that $\rho$ is nonnegative then the positivity of $\gamma$ implies that $\rho$ is greater than $\gamma / \beta$. In this case for $x_{l}=0, \lambda>0$ and then $\eta$ is negative, whereas if $\eta=0$, then $x_{l}>0$.

Finally let $\beta=0$, then $x_{l}=0$ leads to positivity of $\lambda$ and then negativity of $\eta$. On the other hand $\eta=0$ yields $x_{l}=1>0$ and finally part 2) holds. Uniqueness of the solution follows from strong convexity of the objective function and full rank of the constraint.

In the following; we denote the primal-dual solution of Problem 3 by $\left(\hat{\mathbf{X}}^{*}, \hat{\lambda}, \hat{\mu}, \hat{\eta}\right)$; and $\hat{\sigma}^{2}(\rho)$ as the optimal variance of the problem.

Now consider a portfolio that contains $n$ risky assets, $x_{f}$ and $x_{l}$. Let

$$
\hat{\mathbf{X}}_{1}=\left(\mathbf{X}^{\prime}, x_{l}, x_{f}\right)^{\prime}=\left(\hat{\mathbf{X}}^{\prime}, x_{f}\right)^{\prime} \in R^{n+2}
$$

denotes the portfolio. With these assumptions Problem 3 has a new version as follows:

Problem 4.

$$
\begin{gathered}
\min _{\mathbf{X}, x_{l}, x_{f}} \frac{1}{2} \mathbf{X}_{1}^{\prime} \Sigma_{1}^{*} \mathbf{X}_{1} \\
\text { s.t. } \quad \mathbf{1}_{2^{\prime}} \hat{\mathbf{X}}_{1}=\mathbf{1}_{1}^{\prime} \mathbf{X}_{1}+x_{l}=1, x_{l} \geq 0 \\
\overline{\mathbf{U}}_{1}^{\prime} \mathbf{X}_{1}=\rho,
\end{gathered}
$$

where $\mathbf{1}_{2}$ is a $(n+2)$-column vector of ones. We display the primal-dual solution of Problem 4 by $\hat{\mathbf{X}}_{1}^{*}, \hat{\lambda}_{s}, \hat{\mu}_{s}, \hat{\eta}_{s}$.

\footnotetext{
${ }^{4}$ Explicitly we suppose that $\mathrm{u}$ is a constant.
}

Corollary 3.8. Problem 4 has the following unique primal-dual solution:

1) If $s>-\beta / u$, then

a) For $\rho>\gamma_{s} / \beta_{s}, \quad x_{l}=0$ and $\hat{\eta}_{s}=-\hat{\lambda}_{s}>0$ and $\mathbf{X}, x_{f}, \hat{\lambda}_{s}, \hat{\mu}_{s}$ are identical to the primal-dual solution of Problem 2.

b) For $\rho \leq \gamma_{s} / \beta_{s}$,

$$
\begin{aligned}
& \mathbf{X}=\hat{\mu}_{s} \Sigma^{*-1} \overline{\mathbf{U}}, x_{l}=1-\hat{\mu}_{s} \beta_{s}, x_{f}=s u \hat{\mu}_{s}, \\
& \hat{\mu}_{s}=\frac{\rho}{\gamma_{s}}, \hat{\eta}_{s}=\hat{\lambda}_{s}=0 .
\end{aligned}
$$

2) If $s \leq-\beta / u$, then

$$
\begin{aligned}
& \mathbf{X}=\hat{\mu}_{s} \Sigma^{*-1} \overline{\mathbf{U}}, x_{l}=1-\hat{\mu}_{s} \beta_{s}, x_{f}=s u \hat{\mu}_{s}, \\
& \hat{\mu}_{s}=\frac{\rho}{\gamma_{s}}, \hat{\eta}_{s}=\hat{\lambda}_{s}=0 .
\end{aligned}
$$

Proof. Rearrange the portfolio $\hat{\mathbf{X}}_{1}$ to $\left(\mathbf{X}_{1^{\prime}}, x_{l}\right)^{\prime}$, where $\mathbf{X}_{1}=\left(\mathbf{X}^{\prime}, x_{f}\right)^{\prime}$. Now apply Theorem 3.7 for the portfolio $\left(\mathrm{X}_{1^{\prime}}, x_{l}\right)^{\prime}$ and use the definition of $\beta_{1}$.

In order to apply Corollary 3.8 , specification of sign of $\beta_{s}$ is necessary. The value of $\beta$ has the main role in determining the sign of $\beta_{s}$. For example when $\beta \geq 0$, only part 1 ) of Corollary 3.8 gives the answer, whereas for negative $\beta$, both parts of the Corollary can be applied.

In the following let

$$
\left(\left(\hat{\mathbf{X}}_{0}^{* \prime}, x_{l_{0}}^{*}, \hat{x}_{f_{0}}^{*}\right)^{\prime}, \hat{\lambda}_{0}, \hat{\mu}_{0}, \hat{\eta}_{0}\right)
$$

denote the primal-dual solution of Problem 4 when $\sigma_{f}^{2}=0$ which are stated as follows:

Proposition 3.9. If $\rho>u$, then $x_{l_{0}}^{*}=0$ and $\hat{\eta}_{0}=-\hat{\lambda}_{0}>0$ and otherwise

$$
\left(\hat{\mathbf{X}}_{0}^{*}, \hat{x}_{f_{0}}^{*}\right)^{\prime},
$$

$\hat{\lambda}_{0}$ and $\hat{\mu}_{0}$ is identical to the primal-dual solution of Problem 2, when $\sigma_{f}^{2}=0$. Also for $\rho \leq u$,

$$
\begin{aligned}
& \hat{\mathbf{X}}_{0}^{* \prime}=0, \quad x_{l_{0}}^{*}=1-\frac{\rho}{u}, \\
& \hat{x}_{f_{0}}^{*}=\frac{\rho}{u}, \quad \hat{\lambda}_{0}=\hat{\mu}_{0}=\hat{\eta}_{0}=0 .
\end{aligned}
$$

Proof. See Theorem 1.12 of [6].

We show the optimal variance of Problem 4 when $\sigma_{f}^{2}=0$ by $\hat{\sigma}_{0}^{2}(\rho)$ that is

$$
\hat{\sigma}_{0}^{2}(\rho)= \begin{cases}0, & \text { if } \rho \leq u \\ (\rho-u)^{2} / \delta_{f}, & \text { if } \rho>u .\end{cases}
$$

Theorem 3.10. The primal-dual solution and optimal variance of the Problem 4 are continuous with respect to $\sigma_{f}^{2} \in[0, \infty){ }^{4}$

Proof. Define the function $g$ by 


$$
g(t)=\frac{t \gamma+u^{2}}{t \beta+u}, \quad t \in R .
$$

Observe that for any $\sigma_{f}^{2} \in(0, \infty), g\left(\sigma_{f}^{2}\right)=\gamma_{s} / \beta_{s}$.

Let $\beta<0$, then $g$ is strictly increasing on the interval $(-\infty,-u / \beta)$, and hence it is one to one. Also, $u<\gamma_{s} / \beta_{s}$ (see Figure 1). We fix $\rho$ and investigate the claim.

Suppose $\rho \leq u$ and $\sigma_{f}^{2} \in(0, \infty)$. By Corollary 3.8 the primal-dual solution related to any $s=\left(\sigma_{f}^{2}\right)^{-1}$ is

$$
\begin{gathered}
\mathbf{X}_{s}=\hat{\mu}_{s} \Sigma^{*-1} \overline{\mathbf{U}}, x_{l_{s}}=1-\hat{\mu}_{s} \beta_{s}, x_{f_{s}}=s u \hat{\mu}_{s}, \\
\hat{\mu}_{s}=\frac{\rho}{\gamma_{s}}, \hat{\eta}_{s}=\hat{\lambda}_{s}=0
\end{gathered}
$$

and optimal variance is

$$
\hat{\sigma}_{s}^{2}(\rho)=\frac{\rho^{2}}{\gamma_{s}}=\rho \hat{\mu}_{s} .
$$

Because of the positivity of $\gamma_{s}, \hat{\mu}_{s}$ and consequently each of the above terms are continuous with respect to $\sigma_{f}^{2} \in(0, \infty)$. By taking limit of these terms when $\sigma_{f}^{2}$ goes to zero (or equivalently when $S$ goes infinity) and comparing the results with Equations (18) and (19) the assertion holds.

Now let $\rho>u$ and $\sigma_{f}^{2} \in(0, \infty)$. Note that

$$
g^{-1}(\rho)<-u / \beta \text {. }
$$

By Corollary 3.8 the primal-dual solution is

$$
\begin{gathered}
\hat{\eta}_{s}=-\hat{\lambda}_{s}=\left\{\begin{array}{ll}
\left(\beta_{s} \rho-\gamma_{s}\right) / \delta_{s}, & \text { if } \sigma_{f}^{2}<g^{-1}(\rho) \\
0, & \text { if } \sigma_{f}^{2} \geq g^{-1}(\rho)
\end{array},\right. \\
\hat{\mu}_{s}= \begin{cases}\left(\alpha_{s} \rho-\beta_{s}\right) / \delta_{s}, & \text { if } \sigma_{f}^{2}<g^{-1}(\rho) \\
\rho / \gamma_{s}, & \text { if } \sigma_{f}^{2} \geq g^{-1}(\rho)\end{cases} \\
x_{l_{s}}= \begin{cases}0, & \text { if } \sigma_{f}^{2}<g^{-1}(\rho) \\
1-\hat{\mu}_{s} \beta_{s}, & \text { if } \sigma_{f}^{2} \geq g^{-1}(\rho)\end{cases}
\end{gathered}
$$

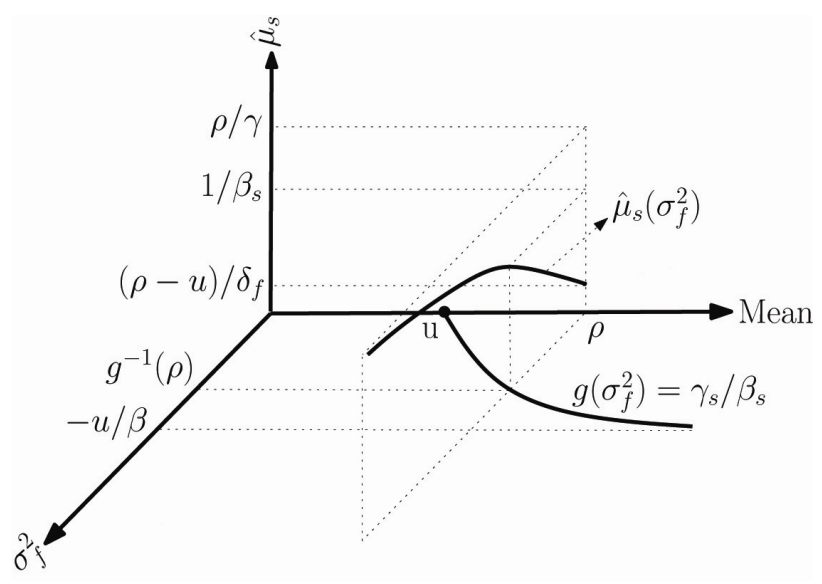

Figure 1 . The continuity of $\hat{\mu}_{\mathrm{s}}$ when $\boldsymbol{\beta}<\mathbf{0}$.

$$
\begin{gathered}
x_{f_{s}}= \begin{cases}s\left(\hat{\lambda}_{s}+u \hat{\mu}_{s}\right), & \text { if } \sigma_{f}^{2}<g^{-1}(\rho) \\
s u \hat{\mu}_{s}, & \text { if } \sigma_{f}^{2} \geq g^{-1}(\rho)\end{cases} \\
\mathbf{X}_{s}= \begin{cases}\Sigma^{*-1}\left(\hat{\lambda}_{s} \mathbf{1}+\hat{\mu}_{s} \overline{\mathbf{U}}\right), & \text { if } \sigma_{f}^{2}<g^{-1}(\rho) \\
\hat{\mu}_{s} \Sigma^{*-1} \overline{\mathbf{U}}, & \text { if } \sigma_{f}^{2} \geq g^{-1}(\rho)\end{cases}
\end{gathered}
$$

and finally the optimal variance is

$$
\hat{\sigma}_{s}^{2}(\rho)=\left\{\begin{array}{ll}
\left(\alpha_{s} \rho^{2}-2 \beta_{s} \rho+\gamma_{s}\right) / \delta_{s}, & \text { if } \sigma_{f}^{2}<g^{-1}(\rho) \\
\rho^{2} / \gamma_{s}, & \text { if } \sigma_{f}^{2} \geq g^{-1}(\rho)
\end{array} .\right.
$$

As we know $\gamma_{s}$ is positive. thus the primal-dual solution and optimal variance are continuous on

$$
\left(g^{-1}(\rho), \infty\right)
$$

Also, it is easily seen that two parts of all of the above terms coincide at $\sigma_{f}^{2}=g^{-1}(\rho)$. Now the assertion holds by Theorem 3.5 and in this case, the proof is complete.

If $\beta=0$, then for $\rho \leq u$, part (1).(b) of Corollary 3.8 holds. Also for $\rho>u$, conditions (20)-(25) hold.

When $\beta$ is positive the proof can be separated into two parts, $u \leq \gamma / \beta$ or $u>\gamma / \beta$.

In the first case for $\rho \leq u$, part 1) b) of Corollary 3.8 holds and for $\rho \leq \gamma / \beta$, only part 1) a) of Corollary 3.8 holds. For $u<\rho<\gamma / \beta$, the primal-dual solution and optimal variance are those which have stated in Equations (20)-(25). Finally in the second case for $\rho \leq \gamma / \beta$ and $\rho \geq u$, part 1) a) and part 1) b) of Corollary 3.8 hold, respectively. When $\gamma / \beta<\rho<u$, Equations (20)-(25) hold but with a change in two conditions of each equation. In all cases it is easy to investigate the continuity of primal-dual solution and optimal variance with respect to $\sigma_{f}^{2}$ on the interval $[0, \infty)$. This completes the proof.

Like Corollary 3.6 we can state the following corollary:

Corollary 3.11 Let the portfolio of Problem 3 contains a cash account $x_{c}$ for which its rate of increments $\lambda_{c}$ is uncorrelated with the total-return of the other securities. If the variance of $\lambda_{c}$ converges to zero, then the primal-dual and the optimal variance of Problem 3 converges to the same values for which $x_{c}$ is replaced by a riskless cash account $x_{c}$ with the same mean total-return.

\section{Co-Mean Total-Return Free Securities}

In the previous section, we investigate the case in which the portfolio contains a free security. In this section we consider a portfolio with more than one free securities $x_{f_{1}}, \cdots, x_{f_{i}}$ which have the same mean total-return $u$. We call such free securities co-mean total-return or briefly 
co-mean free securities. Also we denote the vector weight of co-mean free securities by

$$
\dot{\mathbf{X}}_{i}:=\left(x_{f_{1}}, \cdots, x_{f_{i}}\right)^{\prime}
$$

and call it and its portion in the primal solution, the "free part" of the portfolio and the "free solution", respectively. In the following, let

$$
s_{j}:=\left(\sigma_{f_{j}}^{2}\right)^{-1} \text { for } j=1, \cdots, i .
$$

\subsection{Portfolio with Risky and Co-Mean Free Securities}

In this section we assume that the portfolio is

$$
\mathbf{X}_{i}:=\left(\mathbf{X}^{\prime}, \dot{\mathbf{X}}_{i^{\prime}}\right)^{\prime} \in R^{n+i}
$$

where $\mathbf{X}$ and $\dot{\mathbf{X}}_{i}$ are the risky and the free part of the portfolio, respectively. Let $\overline{\mathbf{U}}_{i}$ and $\Sigma_{i}^{*}$ are the mean vector and the covariance matrix of total-returns of securities, respectively, and $\mathbf{1}_{i}$ is an $(n+i)$-column vector of ones. So the portfolio selection problem is:

\section{Problem 2(i).}

$$
\begin{gathered}
\min _{\mathbf{X}_{i}} \frac{1}{2} \mathbf{X}_{i^{\prime}} \Sigma_{i}^{*} \mathbf{X}_{i} \\
\text { s.t. } \quad \mathbf{1}_{i^{\prime}} \mathbf{X}_{i}=1, \\
\overline{\mathbf{U}}_{i^{\prime}} \mathbf{X}_{i}=\rho .
\end{gathered}
$$

Note that Problems 2(1) an 2 are equivalent. The assumptions are the same as those stated in section 2.1, namely (A.1) and (A.3). As before, we define the following constants:

$$
\alpha_{i}:=\mathbf{1}_{i^{\prime}} \Sigma_{i}^{*-1} \mathbf{1}_{i}, \beta_{i}:=\mathbf{1}_{i^{\prime}} \Sigma_{i}^{*-1} \overline{\mathbf{U}}_{i}, \gamma_{i}:=\overline{\mathbf{U}}_{i^{\prime}} \Sigma_{i}^{*-1} \overline{\mathbf{U}}_{i},
$$

and

$$
\delta_{i}:=\alpha_{i} \gamma_{i}-\beta_{i}^{2}, \quad \delta_{f}^{(i)}:=\alpha_{i} r^{2}-2 \beta_{i} r+\gamma_{i} .
$$

In the following $\left(\mathbf{X}_{i}^{*}, \lambda_{i}, \mu_{i}\right)$ and $\sigma_{i}^{2}(\rho)$ denote the primal-dual solution and the optimal variance of Problem 2(i), respectively.

Lemma 4.1. Let $\alpha, \beta, \gamma, \delta$ and $\delta_{f}$ be the constants related to the risky part of the portfolio, then

1) $\alpha_{i}=\alpha+s_{1}+\ldots+s_{i}$,

2) $\beta_{i}=\beta+\left(s_{1}+\cdots+s_{i}\right) u$,

3) $\gamma_{i}=\gamma+\left(s_{1}+\cdots+s_{i}\right) u^{2}$,

4) $\delta_{f}^{(i)}=\delta_{f}$,

5) $\delta_{i}=\delta+\left(s_{1}+\cdots+s_{i}\right) \delta_{f}$,

Proof. The proof is obvious.

We mention that two portfolio selection problems are equivalent, if they have the same optimal variance for any desired mean return $\rho$ for the portfolio. Equivalently this means that their efficient frontiers coincide.

Proposition 4.2. Let $j<i$. Problem 2(i) with the set of co-mean free securities

$$
\left\{x_{f_{1}}, \cdots, x_{f_{i}}\right\}
$$

and Problem 2(j) with the set of co-mean free securities

$$
\left\{x_{f_{1}}, \cdots, x_{f_{j-1}}, x_{f^{*}}\right\}
$$

(for $j=1$ this set contains $x_{f^{*}}$ only) are equivalent, where

$$
S^{*}:=\left(\sigma_{f^{*}}^{2}\right)^{-1}=\sum_{k=j}^{i} S_{k} .
$$

Precisely, in both problems dual solutions are identical and in primal solutions, individual weights of common securities are identical and

$$
x_{f^{*}}=\sum_{k=j}^{i} x_{f_{k}} .
$$

Proof. Considering Lemma 4.1 and the simple fact that

$$
\sum_{k=1}^{i} s_{k}=\sum_{k=1}^{j-1} s_{k}+s^{*},
$$

Theorem 2.5 shows that both problems have the same dual solutions, say $\lambda_{i}$ and $\mu_{i}$, and consequently have the same optimal variance. To complete the proof let

$$
\mathbf{X}_{i}^{*}=\left(\mathbf{X}^{\prime}, x_{f_{1}} \cdots, x_{f_{i}}\right)^{\prime} .
$$

Then

$$
x_{f_{k}}=s_{k}\left(\lambda_{i}+\mu_{i} u\right)
$$

for $k=1, \cdots, i$. Now

$$
\sum_{k=j}^{i} x_{f_{k}}=\sum_{k=j}^{i} s_{k}\left(\lambda_{i}+\mu_{i} u\right)=s^{*}\left(\lambda_{i}+u \mu_{i}\right)
$$

which is the optimal weight of the last free asset in Problem 2(j).

Proposition 4.3. Problem 2(i) and Problem 2(1) are equivalent if $\sigma_{f_{1}}^{2}=0$. Precisely, no free security appears in the primal solution of Problem 2(i), i.e.

$$
\left(x_{f_{2}}, \cdots, x_{f_{i}}\right)=(0, \cdots, 0),
$$

and both problems have identical primal-dual solution.

Proof. Let $\sigma_{f_{1}}^{2}=0$. By Theorem 3.4, for $i=2, \cdots, n$, each free security has the following weight in the solution of Problem 2(i):

$$
x_{f_{i}}=s_{i}(\lambda+u \mu)
$$

where $\lambda=-u \mu$. Now the assertion holds.

Theorem 4.4. The risky and the dual solutions, and the optimal variance of Problem 2(i) converge to that of 
Problem 2 when $\sigma_{f}^{2}=0$, if

$$
s^{*-1}=\left(\sum_{k=1}^{i} s_{k}\right)^{-1}
$$

converges to zero. Besides, the sum of components of the free solution converges to $x_{f_{0}}^{*}$.

Proof. Let $j=1$ in Proposition 4.2 and construct the dummy portfolio

$$
\mathbf{X}_{1}=\left(\mathbf{X}^{\prime}, x_{f^{*}}\right)^{\prime},
$$

where

$$
\mathbb{E}\left(u_{f^{*}}\right)=u
$$

Now the assertion follows from Proposition 4.2 and Theorem 3.5.

Note. As indicated in Theorem 4.4, it is seen that

$$
\sigma_{f^{*}}^{2}=\frac{1}{1 / \sigma_{f_{1}}^{2}+\cdots+1 / \sigma_{f_{i}}^{2}} .
$$

Obviously if more and more co-mean free securities with low variances are added to the portfolio, then $\sigma_{f^{*}}^{2}$ is more and more close to zero (this is the advantage of a set of co-mean free securities with respect to one free security). Consequently the portfolio selection problem becomes equivalent to the problem in which the set of co-mean free securities are replaced by a riskless security with that total-return in the limit sense.

\subsection{Portfolio with Risky and Co-Mean Free Securities and a Guaranteed Total Loss}

In this section, we investigate the effect of the presence of co-mean free securities in portfolio selection when portfolio consists of $n \geq 1$ risky securities and a total loss security. As before let $\mathbf{X}$ and $\dot{\mathbf{X}}_{i}$ be the risky and the free part of the portfolio, respectively. Now the portfolio is

$$
\hat{\mathbf{X}}_{i}:=\left(\mathbf{X}^{\prime}, x_{l}, \dot{\mathbf{X}}_{i^{\prime}}\right)^{\prime}=\left(\hat{\mathbf{X}}^{\prime}, \dot{\mathbf{X}}_{i^{\prime}}\right) \in R^{n+i+1}
$$

and the problem can be stated as:

Problem 4(i).

$$
\begin{gathered}
\min _{\hat{\mathbf{X}}_{i}} \frac{1}{2} \mathbf{X}_{i^{\prime}} \Sigma_{i}^{*} \mathbf{X}_{i} \\
\text { s.t. } \quad \mathbf{1}_{i+1} \hat{\mathbf{X}}_{i}=\mathbf{1}_{i^{\prime}} \mathbf{X}_{i}+x_{l}=1, x_{l} \geq 0 \\
\overline{\mathbf{U}}_{i^{\prime}} \mathbf{X}_{i}=\rho,
\end{gathered}
$$

where $\mathbf{1}_{i+1}$ is a $(n+i+1)$-column vector of ones. Note that Problems $4(1)$ and 4 are equivalent. We display the primal-dual solution and the optimal variance of Problem $4(i)$ by $\left(\hat{\mathbf{X}}_{i}^{*}, \hat{\lambda}_{i}, \hat{\mu}_{i}, \hat{\eta}_{i}\right)$ and $\hat{\sigma}_{i}^{2}(\rho)$, respectively.

Theorem 4.5. Let $j<i$. Problem 4(i) with the set of free securities

$$
\left\{x_{f_{1}}, \cdots, x_{f_{i}}\right\}
$$

and Problem 4(j) with the set of free securities

$$
\left\{x_{f_{1}}, \cdots, x_{f_{j-1}}, x_{f^{*}}\right\}
$$

(when $j=1$, the set contains only $x_{f^{*}}$ ) are equivalent, where

$$
s^{*}:=\left(\sigma_{f^{*}}^{2}\right)^{-1}=\sum_{k=j}^{i} s_{k} .
$$

Precisely, in both problems dual solutions are identical and in primal solutions, individual weights of common securities are identical and

$$
x_{f^{*}}=\sum_{k=j}^{i} x_{f_{k}} .
$$

Proof. Let

$$
\hat{\mathbf{X}}_{i}^{*}=\left(\mathbf{X}^{\prime}, x_{l}, x_{f_{1}} \cdots, x_{f_{i}}\right)^{\prime} .
$$

If $\beta_{i}>0$ and $\rho \leq \gamma_{i} / \beta_{i}$, then by Theorem 3.7, we have

$$
\begin{gathered}
\mathbf{X}=\hat{\mu}_{i} \Sigma^{*-1} \overline{\mathbf{U}}, \quad x_{l}=1-\hat{\mu}_{i} \beta, \\
\left(x_{f_{1}} \cdots, x_{f_{i}}\right)=u \hat{\mu}_{i}\left(s_{1}, \cdots, s_{i}\right), \\
\hat{\mu}_{i}=\frac{\rho}{\gamma_{i}}, \quad \hat{\eta}_{i}=\hat{\lambda}_{i}=0,
\end{gathered}
$$

and

$$
\hat{\sigma}_{i}^{2}(\rho)=\frac{\rho^{2}}{\gamma_{i}} .
$$

It can be easily seen that by Lemma 4.1 and the simple fact that

$$
\sum_{k=1}^{i} s_{k}=\sum_{k=1}^{j-1} s_{k}+s^{*},
$$

we have $\beta_{j}=\beta_{i}$ and $\gamma_{j}=\gamma_{i}$. Now the proof is straight forward by part 1) b) of Theorem 3.7. For instance in primal solution of Problem 4(j)

$$
x_{f^{*}}=s^{*} u \hat{\mu}_{j}=s^{*} u \hat{\mu}_{i}=\sum_{k=j}^{i} s_{k} u \hat{\mu}_{i}=\sum_{k=j}^{i} x_{f_{k}} .
$$

The claim can be proved similarly for $\beta_{i} \leq 0$.

If $\beta_{i}=\beta_{j}>0$ and $\rho>\gamma_{i} / \beta_{i}=\gamma_{j} / \beta_{j}$, the assertion follows from part 1) a) of Theorem 3.7 applied to Problems 4(i) and 4(j) and Proposition 4.2.

Theorem 4.6. Problem 4(i) and Problem 4(1) are equivalent if $\sigma_{f_{1}}^{2}=0$. Precisely, no free security appears in the primal solution of Problem 4(i), i.e.

$$
\left(x_{f_{2}}, \cdots, x_{f_{i}}\right)=(0, \cdots, 0) \text {, }
$$

and both problems have the identical primal-dual 
solution.

Proof. The case $i=1$ is trivial. Let $i>1$. Rearrange the portfolio of Problem 4(i), $\hat{\mathbf{X}}_{i}$, to

$$
\left(\left(\mathbf{X}^{\prime}, x_{f_{2}}, \cdots, x_{f_{i}}\right), x_{l}, x_{f_{1}}\right)^{\prime} .
$$

If $\rho \leq u$, then by applying Proposition 3.9 for Problem 4(i), we see that the primal and dual solutions are as follows:

$$
\begin{gathered}
\mathbf{X}=0,\left(x_{f_{2}}, \cdots, x_{f_{i}}\right)=(0, \cdots, 0), \\
x_{l}=1-\frac{\rho}{u}, \quad x_{f_{1}}=\frac{\rho}{u}, \\
\hat{\lambda}_{i}=\hat{\mu}_{i}=\hat{\eta}_{i}=0 .
\end{gathered}
$$

In this case the optimal variance is zero and this complete the proof for $\rho \leq u$.

If $\rho>u$, then by Proposition 3.9, $x_{l}=0$ and $\hat{\eta}_{i}=-\hat{\lambda}_{i}>0$ and otherwise the primal-dual solution is identical to the primal-dual solution of Problem 2(i) when $\sigma_{f_{1}}^{2}=0$. On the other hand, by Theorem 4.3, the primal-dual solution is identical to the primal-dual solution of Problem 2(1). Now the assertion follows from Proposition 3.9.

Theorem 4.7. The risky, the total loss and the dual solutions, and the optimal variance of Problem 4(i) converge to that of Problem 4 when $\sigma_{f}^{2}=0$, if

$$
\left(s^{*}\right)^{-1}=\left(\sum_{k=1}^{i} s_{k}\right)^{-1}
$$

converges to zero. Besides, the sum of components of the free solution converges to $x_{f_{0}}^{*}$

Proof. Let $j=1$ in Theorem 4.5 and construct the dummy portfolio

$$
\hat{\mathbf{X}}_{1}=\left(\mathbf{X}^{\prime}, x_{l}, x_{f^{*}}\right)^{\prime},
$$

where

$$
\mathbb{E}\left(u_{f^{*}}\right)=u
$$

Now the assertion holds by Theorems 4.5 and Theorem 3.10 .

Note. Theorems $3.5,3.10,4.4$ and 4.7 state that a free security or a set of co-mean free securities which their total-returns have very low variances and the same mean with the riskless security, can be considered as a alternative for the riskless security.

\section{REFERENCES}

[1] H. M. Markowitz, "Portfolio Selection," The Journal of Finance, Vol. 7, No. 1, 1952, pp. 77-91.

[2] J. Tobin, "Liquidity Preference as a Behavior towards Risk, Review of Economic Studies," Vol. 25, No. 2, 1958, pp. 65-86. doi:10.2307/2296205

[3] R. H. Tütüncü, "A Note on Calculating the Optimal Risky Portfolio," Finance and Stochastics, Vol. 5, No. 3, 2001, pp. 413-417. doi:10.1007/PL00013542

[4] R. C. Merton, "An Analytic Derivation of the Efficient Portfolio Frontier," Journal of Financial and Quantitative Analysis, Vol. 7, No. 4, 1972, pp. 1851-1872. doi: $10.2307 / 2329621$

[5] R. Roll, "A Critique of the Asset Pricing Theory's Tests. Part I. On Past and Potential Testability of the Theory," Journal of Financial Economics, Vol. 4, No. 2, 1997, pp. 129-176. doi:10.1016/0304-405X(77)90009-5

[6] M. C. Steinbach, "Markowitz Revisited: Mean-Variance Models in Financial Portfolio Analysis," Siam Review, Vol. 43, No. 1, 2011, pp. 31-85. doi: $10.1137 / \mathrm{S} 0036144500376650$ 